\title{
Forecast of Solar Proton Events with nOAa Scales Based on Solar X-Ray Flare Data Using NeUral Network
}

\author{
Eui-Jun JeOng ${ }^{1}$, Jin-Yi LeE ${ }^{1}$, Yong-JAe Moon ${ }^{1,2}$, And Jongyeop PARK ${ }^{2}$ \\ ${ }^{1}$ Department of Astronomy and Space Science, Kyung Hee University, Yongin 446-701, Korea \\ ejjeong@khu.ac.kr, jlee@khu.ac.kr, moonyj@khu.ac.kr \\ ${ }^{2}$ School of Space Research, Kyung Hee University, Yongin 446-701, Korea
}

Received May 1, 2014; accepted August 8, 2014

\begin{abstract}
In this study we develop a set of solar proton event (SPE) forecast models with NOAA scales by Multi Layer Perceptron (MLP), one of neural network methods, using GOES solar X-ray flare data from 1976 to 2011. Our MLP models are the first attempt to forecast the SPE scales by the neural network method. The combinations of X-ray flare class, impulsive time, and location are used for input data. For this study we make a number of trials by changing the number of layers and nodes as well as combinations of the input data. To find the best model, we use the summation of F-scores weighted by SPE scales, where F-score is the harmonic mean of PODy (recall) and precision (positive predictive value), in order to minimize both misses and false alarms. We find that the MLP models are much better than the multiple linear regression model and one layer MLP model gives the best result.
\end{abstract}

Key words: space weather - solar proton event - solar X-ray flare - neural network - multi layer perceptron

\section{INTRODUCTION}

Solar proton event (SPE), which is defined as solar energetic particle events having a flux of $>10 \mathrm{MeV}$ protons greater than or equal to 10 particle flux unit (pfu, number of particles $\mathrm{cm}^{-2} \mathrm{sec}^{-1} \mathrm{ster}^{-1}$ ), is one of the strongest solar activities. The SPE is very significant for space weather in that it can seriously affect hardware in satellites and astronauts. It has been well known that SPEs are very intimately associated with solar activities such as solar flares and coronal mass ejections (CMEs). There have been many studies on the relationship between SPE and X-ray flares; e.g., shock acceleration of electrons and ions in solar flares (Ellison et al. 1985), proton events and X-ray flares in the last three solar cycles (Belov et al. 2005), and a study of solar energetic particle events of 1997-2006 (Cane et al. 2010).

Machine learning algorithms such as support vector machine and neural network have been used for the study of solar activities (e.g., Colak \& Qahwaji 2009; Al-Omari et al. 2010; Choi et al. 2012). The algorithms can learn systems from historical data. Therefore, the algorithms make it possible to construct a forecast model even though the relationship between cause and effect is not clearly understood. Neural networks have been broadly used in space weather applications such as the prediction of solar activities (Wang 2000; Gong et al. 2004; Wang et al. 2008; Qahwaji et al. 2007, 2008; Henwood et al. 2010) and geomagnetic activities (Lundstedt 1992; Hernandez et al. 1993; Freeman et al. 1993; Valach et al. 2009; Ji et al. 2013).

SPE prediction models have been developed by neu-

CORRESPONDING AUTHOR: J.-Y. Lee ral networks (Wang 2000; Gong et al. 2004; Cui et al. 2012 ), which are mostly applied to the prediction of SPE occurrence (Yes or No of SPE events). The models used several kinds of input data such as sunspot properties from white light and magnetogram observations, flare, and radio burst. While two models (Gong et al. 2004; Cui et al. 2012) can predict "event" or "no event", Wang (2000) suggested a two-pass model using by both a yes/no event prediction and forecast three levels of SPE events.

In this study we make the first attempt to forecast SPE scales by neural network. We develop a set of SPE forecast models that predicts SPE scales, which represents the SPE flux according to NOAA space weather scales (S), by multi linear regression (MLR) and multi layer perceptron (MLP) methods using X-ray flare information. The MLP is a feedforward artificial neural network model, which is to classify complex patterns. It has been used for an automatic solar flare detection (Qu et al. 2003), a prediction of interplanetary shock arrival time (Turchenko et al. 2007), and a prediction of ozone levels (Wang et al. 2006).

This work would be a part of a two stage of SPE forecast models: SPE probability (Park et al. 2010) for the first stage and SPE scale, which represents the SPE flux according to NOAA space weather scales $(\mathrm{S})$, for the second stage. The first stage is based on Park et al. (2010) who developed a SPE prediction model using X-ray flare information based on the dependence of SPEs on their associated flares. In this study, as the second stage, we develop a forecast model to predict SPE scales using the neural network method.

We use X-ray flare class, location (longitude, latitude), and impulsive time (flare peak time - flare start 
Table 1

Number of SPE events used in data selection

\begin{tabular}{lc}
\hline Data Sets & The Number of Data \\
\hline SPEs (SWPC Report) & 246 \\
associated flares (stronger or equal to X-ray class M) & 203 \\
associated flares (availability of time information) & 175 \\
SPEs for training (from 1976 to 1996) & 105 \\
SPEs for prediction (from 1997 to 2011) & 70 \\
\hline
\end{tabular}

Table 2

Input and output data

\begin{tabular}{lll}
\hline \multicolumn{2}{l}{ Input (flare information) } & Output $\left(\mathrm{SPE}\right.$ scale) ${ }^{\mathrm{c}}$ \\
\hline X-ray flare class & case 1. 3 for $\mathrm{M} i^{\mathrm{a}}$ class and 4 for X $i$ class & $\mathrm{S} 1: 10 \leq \mathrm{flux}^{\mathrm{b}}<10^{2}$ \\
& case 2. $i$ for $\mathrm{M} i$ and $10+i$ for X $i$ class & $\mathrm{S} 2: 10^{2} \leq \mathrm{flux}<10^{3}$ \\
Flare location & $0 \sim 90$ (center of the Sun: 90, limb: 0) & $\mathrm{S} 3: 10^{3} \leq \mathrm{flux}<10^{4}$ \\
Impulsive time & flare peak time - flare start time(minute) & $\mathrm{S} 4: 10^{4} \leq$ flux $<10^{5}$ \\
\hline
\end{tabular}

a $i$ : intensity of X-ray flare

b flux: flux in pfu (number of particles $\mathrm{cm}^{-2} \mathrm{sec}^{-1} \mathrm{ster}^{-1}$ )

c No S5 (flux $\geq 10^{5}$ ) event during the period used in this analysis

time) as input data. We use SPE scales (S1-S4), which is provided by NOAA space weather prediction center, as output data. We construct the forecast models depending on the number of layers and nodes of the MLP as well as combinations of input data. For the evaluation of the models, we use the F-score, which is the harmonic mean of PODy defined by recall and precision, to find the best models.

This paper is organized as follows. Section 2 describes data, MLR and MLP methods, and statistical verification method. In Section 3, we evaluate and compare 1 MLR model and 4 MLP models. In Section 4, we give a brief summary and conclusion.

\section{DATA AND METHODS}

\subsection{Data}

We use SPE scales in the solar proton event list ${ }^{\mathbf{1}}$ provided by Space Wether Prediction Center of National Oceanic and Atmospheric Administration (NOAA). There are 246 SPEs from April 1976 to September 2011. We select 203 SPEs associated with X-ray flares, which are stronger or equal to $\mathrm{M}$ class $\left(\geq 10^{-5} \mathrm{~W} / \mathrm{m}^{2}\right)$. Then we select 175 SPEs which are associated with flares whose peak/start times and locations are available. From the GOES X-ray flare list ${ }^{2}$ provided by NOAA National Geophysical Data Center, we use Xray flare class, its location, and its impulsive time as input data. The impulsive time is from the flare start to peak time.

Table 1 shows the selected numbers of SPEs for this study from April 1976 to September 2011. We separate the data into two sets for training data from 1976 to 1996 and test data from 1997 to 2011. We preprocess

\footnotetext{
${ }^{1}$ Solar proton events affecting the Earth environment: http: //www.swpc.noaa.gov/ftpdir/indices/SPE.txt

$2_{\text {ftp: / /ftp.ngdc.noaa.gov/STP/SOLAR_DATA/SOLAR_FLARES/ }}$ FLARES_XRAY
}

Table 3

Contingency table between predictions and observations

\begin{tabular}{llll}
\hline & & \multicolumn{2}{c}{ Observation } \\
& & Yes & No \\
\hline Prediction & Yes & a & b \\
& No & c & \\
\hline
\end{tabular}

$\mathrm{a}=$ hit, $\mathrm{b}=$ false alarm, $\mathrm{c}=$ miss

them so that the program can recognize the characteristics of the data easily before we apply the MLR and MLP programs to the data set. The flare locations are given from 0 (limb) to 90 (center of the Sun). We number X-ray class with the following two cases: case 1) 3 for all strengths of $\mathrm{M}$ class and 4 for all strengths of $\mathrm{X}$ class and case 2) an addition of 10 to the strength of $\mathrm{X}$-class flare. For case 2, M5 is 5 and X7 is 17. As output data, we use SPE scales. Table 2 shows the input and output data.

\subsection{MLR and MLP}

We apply MLR and MLP algorithms to solar flare data for the prediction of SPE scales. We use Apache Common Math library ${ }^{\mathbf{3}}$ to preprocess the input data. It is an open library that can be efficiently used in scientific computing. Then we apply the MLR algorithm to the input and output data. The MLR is one of regression analysis, which is the most commonly used techniques in statistics. It can be used to fit a predictive model to an observed data set of values. In this analysis, the MLR equation is expressed as $Y_{i}=\sum_{k=1}^{3} X_{k i} A_{k}+b$. Here $Y_{i}$ is a dependent variable, $X_{k i}$ is an independent variable, $A_{k}$ is k-dimensional parameter, and $b$ is a constant term, where $i$ is an event number. In this analysis, $X_{k i}$ is the input data, which are X-ray class, flare location,

\footnotetext{
$3_{\text {http: //commons . apache.org/math }}$
} 
Table 4

Statistical verification parameters from the contingency table

\begin{tabular}{llc}
\hline Statistical Parameter & Description & Definition \\
\hline PODy or recall & Probability of Detection of Yes observations & $a /(a+c)$ \\
FAR & False Alarm Ratio & $b /(a+b)$ \\
CSI & Critical Success Index & $a /(a+b+c)$ \\
Precision & Positive predictive value & $a /(a+b)$ \\
\hline
\end{tabular}

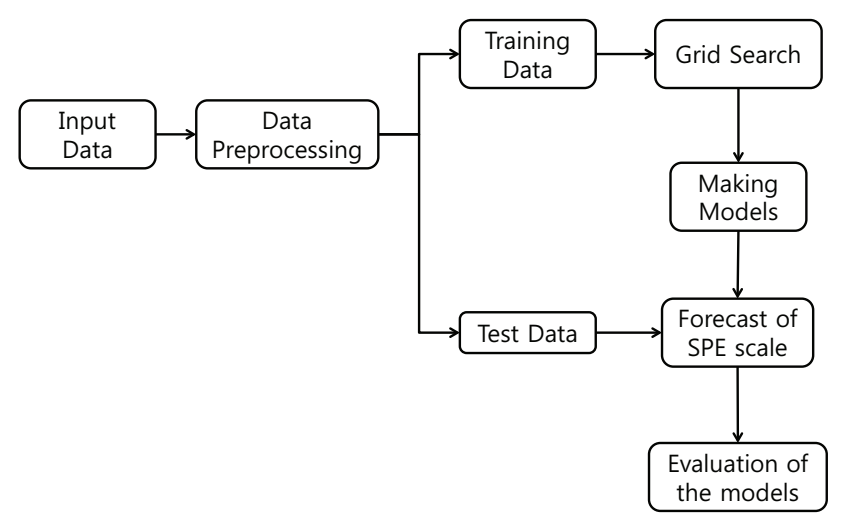

Figure 1. Flow chart of the development of SPE forecast models based on MLP

and impulsive time. And $Y_{i}$ is the SPE scale. To construct the MLR models, first, we find several models with the combinations of input data from the training data set. Second, we obtain output data from the input of test data set.

The MLP is a feedforward artificial neural network model. We use OpenCV ${ }^{4}$ that provides the MLP functions for train and prediction. We train the preprocessed training data with changing the number of layers and nodes. We use a grid-search from 2 to 20 nodes for each layers. In addition, we use a few kernel functions and propagation methods. We use gaussian and sigmoid kernel functions provided by OpenCV for the models. Once after we train the data set, we predict the SPE scale using the test data set by the models. Then we rescale the output obtained from the MLR and MLP models as follows: S1: $0 \leq$ output $<\mathrm{S}_{\min }+\Delta \mathrm{S}, \mathrm{S} 2$ : $\mathrm{S}_{\text {min }}+\Delta \mathrm{S} \leq$ output $<\mathrm{S}_{\text {min }}+2 \times \bar{\Delta} \mathrm{S}, \mathrm{S} 3: \mathrm{S}_{\text {min }}+2 \times \Delta \mathrm{S} \leq$ output $<\mathrm{S}_{\min }+3 \times \Delta \mathrm{S}$, and $\mathrm{S} 4: \mathrm{S}_{\min }+3 \times \Delta \mathrm{S} \leq$ output $\leq$ $\mathrm{S}_{\max }$. Here $\Delta \mathrm{S}$ is defined as $\left(\mathrm{S}_{\max }-\mathrm{S}_{\min }\right) / 4 . \mathrm{S}_{\min }$ and $\mathrm{S}_{\max }$ are the minimum and maximum values of output obtained by each model, respectively. Finally, we compare the output data with the observations for the test data set.

\subsection{Statistical Verification}

We evaluate the performance of the models using statistical parameters. In Table 3 , the " $a$ " is the number of event forecasts that corresponds to the event observations (i.e., the number of hits); the " $b$ " is the number of event forecasts that do not correspond to the observed events (i.e., the number of false alarms); the " $c$ "

\footnotetext{
${ }^{4}$ http: //opencv.org
}

is the number of no-event forecasts corresponding to the observed events (i.e., the number of misses). As an example for the $\mathrm{S} 1$, the 'a' indicates the number of S1 forecasts that corresponds to the S1 observations. ' $b$ ' indicates the number of S1 forecasts that corresponds to the S2-S4 observations.

In Table 4, we summarize various statistical parameters based on the contingency table. PODy is the proportions of hits. FAR is the proportion of incorrect prediction. CSI is the proportion of correct predictions that are either predicted or observed. The PODy is a good verification parameter when we focus on the misses. The CSI considers both misses and false alarms. It is hard to say which parameter is more important in space weather forecast. Therefore, we use a verification parameter, a balanced F-score, which is the harmonic mean of precision and recall. The precision is the probability of observation of 'Yes' predictions (see Table 4) and the recall is the same with the PODy. The balanced $\mathrm{F}$-score is given by

$$
\text { F-score }=\frac{2 * \text { Recall } * \text { Precision }}{\text { Recall }+ \text { Precision }} .
$$

Since the prediction of stronger SPE scales is much more important than that of weaker ones, we use a parameter, summation of F-scores weighted by SPE scale (SF), to see an overall performance of the model for all $\mathrm{SPE}$ scales. The $\mathrm{SF}$ is given by

$$
\mathrm{SF}=\sum_{k=1}^{4} k *(\mathrm{~F}-\mathrm{scor} \mathrm{e})_{k}
$$

where $\mathrm{k}$ is the SPE scale.

\section{RESUlts AND Discussion}

We show one MLR and 4 MLP models in Table 5. The MLR model shown in the Table 5 has the highest SF among MLR models with the combinations of input data. This model uses X (X-ray flare class) and T (impulsive time) with case 1 as its input data. In the case of MLP models, we find that a gaussian function is the best kernel since it shows higher PODy and lower FAR. With the changes of the number of layers and nodes as well as the combinations of the input data, we select 4 MLP models whose SF values are larger than 2.5 in Table 5 . The selected MLP models show that XTL combination (X-ray flare class, impulsive time, and location) with case 2 produces higher SF values than other combinations. 


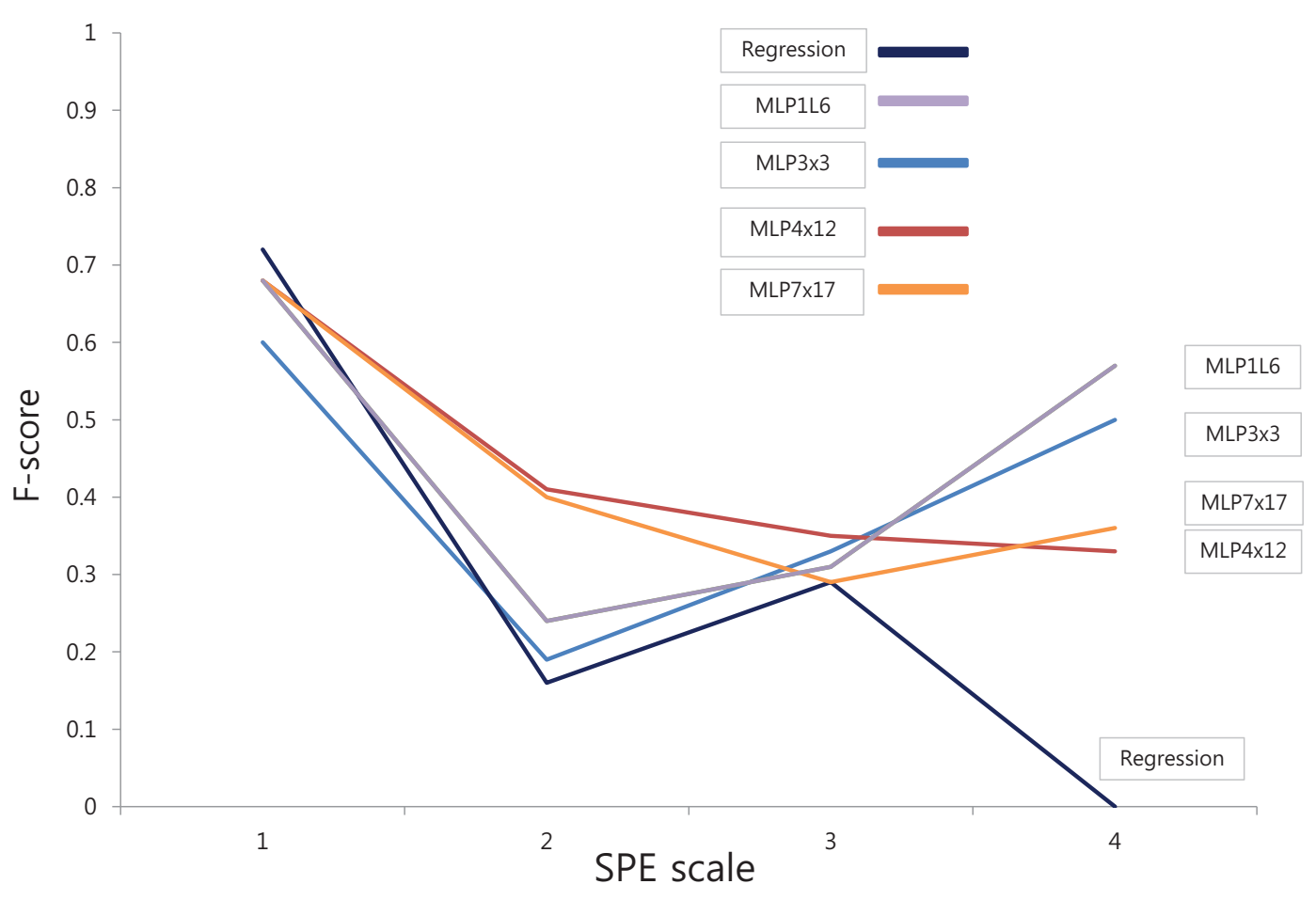

Figure 2. Comparison of the F scores of all five models with SPE scales

Table 5

Kernel functions and parameters of MLP models

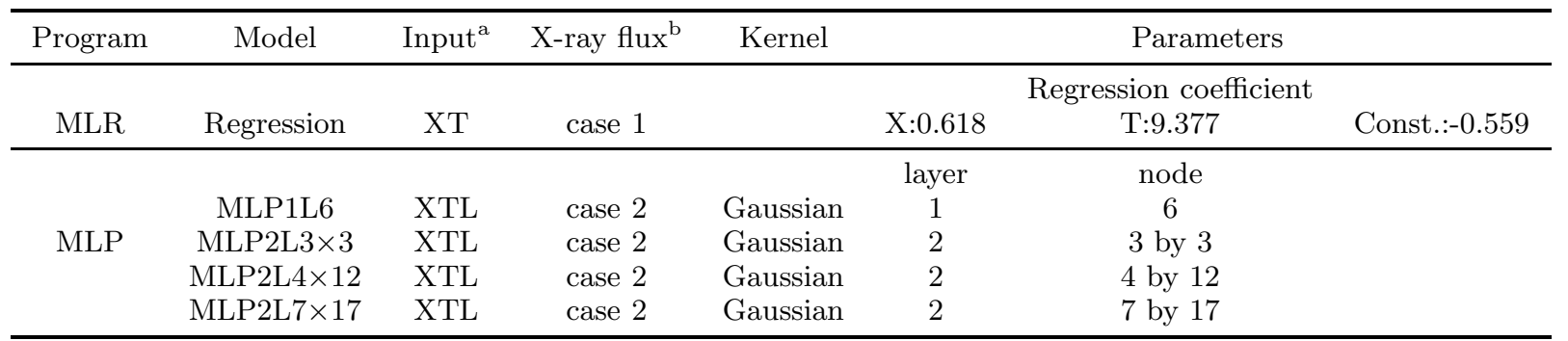

anput: X (X-ray Flare Class), T (Impulsive Time), L (Flare Location)

${ }^{\mathrm{b}} \mathrm{X}$-ray flux : case 1 (All M class: 3 , All X class: 4$)$, case $2($ ex. M5 $=5, \mathrm{X} 7=17)$

Table 6 shows the statistical verification parameters of each model with SPE scales. The MLR model hardly predicts the stronger SPEs than S1. It may come from that the regression method find a model that is more optimized for weaker SPEs since the number of weaker SPEs are much larger than that of stronger SPEs.

We compare the F-scores of all five models with SPE scales in Figure 2. The F-scores of MLP models are much larger than those of the MLR model for the events stronger than S1. In the comparison of the MLP models, the F-score values of MLP1L6 (purple) and MLP2L3 $\times 3$ (blue) for S4 are larger than those of the other models while the F-scores of MLP2L $4 \times 12$ (red) and MLP2L7 $\times 17$ model (orange) for S2 are larger than those of the other models. Especially, MLP1L6 model (purple in Figure 2) has the highest value of F-score, 0.57 for S4. To compare an overall performance of these models, we use the summation of F-scores (SF, Equa- tion (2)) weighted by SPE scale since stronger events are more important in terms of space weather. As a result, we find that the MLP1L6 model has the highest value of SF, 4.37, which is larger than those of the other MLP models. Main advantage of this model is to well predict S4 events, which are extremely strong ones. The values of PODy and CSI also show similar patterns with the F-score values in Table 6. Therefore, we suggest the MLP1L6 model, which has one hidden layer with 6 nodes, for the prediction of SPE scales according to the highest SF.

It is not easy to compare our model with previous other models in that other neural network models mostly predict 'yes' or 'no' of SPE event (Gong et al. 2004; Cui et al. 2012). Wang (2000) used a neural network method for the forecast of three levels of SPE flux. Since his classification are different from our classification and the number of events that he used is too 
Table 6

Statistical verification parameters of all five models with SPE scales

\begin{tabular}{cccccccccc}
\hline Model & Index & Hits & Obs & PODy & FAR & CSI & Precision & F-score & SF \\
\hline \multirow{5}{*}{ Regression } & S1 & 27 & 35 & 0.77 & 0.31 & 0.57 & 0.69 & 0.73 & \\
& S2 & 0 & 20 & 0 & 0 & 0 & 1 & 0.0 & \\
& S3 & 5 & 9 & 0.56 & 0.83 & 0.15 & 0.17 & 0.26 & 2.67 \\
& S4 & 1 & 6 & 0.17 & 0 & 0.17 & 1 & 0.29 & \\
\hline \multirow{5}{*}{ MLP1L6 } & S1 & 27 & 35 & 0.77 & 0.39 & 0.52 & 0.61 & 0.68 & \\
& S2 & 4 & 20 & 0.2 & 0.71 & 0.13 & 0.29 & 0.24 & \\
& S3 & 2 & 9 & 0.22 & 0.5 & 0.18 & 0.5 & 0.31 & 4.37 \\
& S4 & 4 & 6 & 0.67 & 0.5 & 0.4 & 0.5 & 0.57 & \\
\hline \multirow{5}{*}{ MLP2L3×3 } & S1 & 25 & 35 & 0.71 & 0.49 & 0.42 & 0.51 & 0.60 & \\
& S2 & 3 & 20 & 0.15 & 0.75 & 0.1 & 0.25 & 0.19 & \\
& S3 & 2 & 9 & 0.22 & 0.33 & 0.2 & 0.67 & 0.33 & 3.97 \\
& S4 & 3 & 6 & 0.5 & 0.5 & 0.33 & 0.5 & 0.5 & \\
\hline \multirow{5}{*}{ MLP2L4 $\times 12$} & S1 & 26 & 35 & 0.74 & 0.38 & 0.51 & 0.62 & 0.68 & \\
& S2 & 7 & 20 & 0.35 & 0.5 & 0.26 & 0.5 & 0.41 & \\
& S3 & 3 & 9 & 0.33 & 0.63 & 0.21 & 0.38 & 0.35 & 3.87 \\
& S4 & 2 & 6 & 0.33 & 0.67 & 0.2 & 0.33 & 0.33 & \\
\hline \multirow{5}{*}{ MLP2L7×17 } & S1 & 27 & 35 & 0.77 & 0.4 & 0.51 & 0.6 & 0.68 & \\
& S2 & 7 & 20 & 0.35 & 0.53 & 0.25 & 0.47 & 0.40 & \\
& S3 & 2 & 9 & 0.22 & 0.6 & 0.17 & 0.4 & 0.29 & 3.79 \\
& S4 & 2 & 6 & 0.33 & 0.6 & 0.22 & 0.4 & 0.36 & \\
\hline
\end{tabular}

small (14 events), it is difficult to compare his results with ours. On the other hand, several studies to predict SPE flux have been made based on the relationship between SPEs and X-ray flares (Garcia 1994; Balch 2008; Park et al. 2010). We compute the SF value of Park et al.(2010) and find that the value (2.33) is much smaller than those of our neural network models and a little smaller than that of the MLR method.

One may raise a question why MLP1L6 model gives best scores than other models for strong solar proton events and MLP2L4 12 and MLP2L7 17 give best results for weak solar proton events. The neural network algorithms learn systems from historical data, which is useful to construct a forecast model even though the physical relationship between cause and effect is not clearly understood. Therefore, it is hard to find any physical understanding from the neural network models. We examine the dependence of F-scores of the models on the number of nodes and layers. We find that the F-score of a model decreases as the number of layers or nodes. This fact can be understood by the fact that the number of strong solar proton events is much smaller than that of weak solar ones.

\section{Summary AND Conclusion}

We develop the SPE scale forecast models based on solar X-ray flare information (flare class, source location, impulsive time) using the MLR and MLP methods. The models can be used to predict the SPE scale once after a strong flare ( $\mathrm{M}$ or $\mathrm{X}$ class) occurs. For the evaluation of the models, we use several statistical verification parameters such as PODy, CSI, and F-score. To compare an overall performance of the models, we used the SF value, the summation of F-scores weighted by SPE scales.

We find that the statistical verification parameters of the MLP models are much better than the MLR model. A comparison of the SF values shows that the MLP1L6 model of one layer and 6 nodes gives the best result. It is noted that our MLP models are the first attempt to forecast the SPE scales by the neural network method.

As explained in Section 1, we suggest that our model can be a part of a two-stage SPE forecast model: SPE probability forecast (Park et al. 2010; Park et al. 2012) for the first stage and SPE scale forecast (this study) for the second stage. We expect that our models can be also used for the operation of an automatic SPE forecast.

Several studies have shown that SPEs are closely related with CMEs (Kahler et al. 1984; Park et al. 2012; Huang et al. 2012). We plan to develop a model based on both flare and CME parameters as its input data. We will also consider other input data such as hard Xray fluxes (Garcia 2004), magnetic properties(Cui et al. 2012), and other solar activities (Wang 2000; Gong et al. 2004).

\section{ACKNOWLedgments}

This work was supported by the BK21 plus program through the National Research Foundation (NRF) funded by the Ministry of Education of Korea, Basic Science Research Program through the NRF funded by the Ministry of Education (2013R1A1A2058409, NRF-2013R1A1A2012763), and NRF of Korea Grant funded by the Korean Government (NRF-2013M1A3A3A02042232). 


\section{REFERENCES}

Al-Omari, M., Qahwaji, R., Colak, T., \& Ipson, S. 2010, Machine Learning-Based Investigation of the Associations between CMEs and Filaments, Solar Physics, 262, 511

Balch, C. C. 2008, Updated Verification of the Space Weather Prediction Center's Solar Energetic Particle Prediction Model, Space Weather, 6, S01001

Belov, A., Garcia, H., Kurt, V., \& Mavromichalaki, E. 2005, Proton Events and X-Ray Flares in the Last Three Solar Cycles, Cosmic Research, 43, 165

Cane, H. V., Richardson, G., \& von Rosenvinge, T. T. 2010, A Study of Solar Energetic Particle Events of 1997-2006: Their Composition and Associations, JGR, 115, A08101

Ellison, D. C., \& Ramaty, R. 1985, Shock Acceleration of Electrons and Ions in Solar Flares, ApJ, 298, 400

Choi, S. H., Moon, Y.-J., Vien. N. A., \& Park, Y. D. 2012, Application of Support Vector Machine to the Prediction of Geo-Dffective Halo CMEs, JKAS, 43, 125

Colak, T., \& Qahwaji, R. 2009, Automated Solar Activity Prediction: A Hybrid Computer Platform Using Machine Learning and Solar Imaging for Automated Prediction of Solar Flares, Space Weather, Vol. 7, S06001

Cui, Y.-m., Li, R., \& Liu, S.-q. 2012, Short-Term Forecast of Solar Proton Events With Characteristic Physical Quantities of Photospheric Magnetic Fields, Chinese Astronomy and Astrophysics, 36, 169

Freeman, J., Nagai, A., \& Reiff, P. 1993, The Use of Neural Networks to Predict Magnetospheric Parameters for Input to a Magnetospheric Forecast Model, Proceedings of the International Workshop on Artificial Intelligence Applications in Solar-Terrestrial Physics, Lund, Sweden, Sep. 22-24, 1993, 167

Garcia, H. A. 1994, Temperature and Hard X-Ray Signatures for Energetic Proton Events, ApJ, 420, 422

Garcia, H. A. 2004, Forecasting Methods for Occurrence and Magnitude of Proton Storms with Solar Hard X Rays, Space Weather, 2, S06003

Gong, J.-c., Xue, B.-s., Liu, S.-q., Zou, Z.-m., Miao, J., \& Wang, J.-l. 2004, Short-Term Prediction of Solar Proton Events by Neural Network Method, Chinese Astronomy and Astrophysics, 28, 174

Henwood, R., Chapman, S. C., \& Willis, D. M. 2010, Increasing Lifetime of Recurrent Sunspot Groups Within the Greenwich Photoheliographic Results, Solar Physics, 262,299

Hernandez, J. V., Tajima, T., \& Horton, W. 1993, Neural Network Forecasting Geomagnetic Activity, Geophys. Res. Lett., 20, 2707

Huang, X., Wang, H.-N., \& Li, L.-P. 2012, Ensemble Predic- tion Model of Solar Proton Events Associated with Solar Flares and Coronal Mass Ejections, Research in Astronomy and Astrophysics, 12, 313

Ji, E.-Y., Moon, Y.-J., Park, J., \& Lee, D.-H. 2013, Comparison of Neural Network and Support Vector Machine Methods for Kp Forecasting, JGR, 118, 5109

Kahler, S. W., Sheeley, N. R. Jr., Howard, R. A., Michels, D. J., Koomen, M. J., McGuire, R. E., von Rosenvinge, T. T., \& Reames, D. V. 1984, Associations between Coronal Mass Ejections and Solar Energetic Proton Events, JGR, 89,9683

Lundstedt, H. 1992, Neural Networks and Predictions of Solar-Terrestrial Effects, Planet. Space Sci., 40, 457

Park, J., Moon, Y.-J., Lee, D.-H., \& Youn, S. 2010, Dependence of Solar Proton Events on Their Associated Activities: Flare Parameters, JGR, 115, A10, A10105

Park, J., Moon, Y.-J., \& Gopalswamy, N. 2012, Dependence of Solar Proton Events on Their Associated Activities: Coronal Mass Ejection Parameters, JGR, 117, A8, A08108

Qahwaji, R., \& Colak, T. 2007, Automated Short-Solar Flare Prediction Using Machine Learning and Sunspot Associations, Solar Physics, 241, 195

Qahwaji, R., Colak, T., Al-Omari, M., \& Ipson, S. 2008, Automated Prediction of CMEs Using Machine Learning of CME-Flare Associations, Solar Physics, 248, 471

Qu, M., Shih, F. Y., Jing, J., \& Wang, H. 2003, Automated Solar Flare Detection Using MLP, RBF, and SVM, Solar Physics, 217, 157

Turchenko, V., Ternopil, N., Ternopil, D. V., \& Sachenko, A. 2007, Interplanetary Shock Arrival Time Prediction Using Multi-Layer Perceptron, Intelligent Data Acquisition and Advanced Computing Systems: Technology and Applications, 185

Valach, F., Revallo, M., Bochníček, J., \& Hejda, P. 2009, Solar Energetic Particle Flux Enhancement as a Predictor of Geomagnetic Activity in a Neural Network-Based model, Space Weather, 7, S04004

Wang, D., \& Lu, W. Z. 2006, Forecasting Ozone Levels and Analyzing Their Dynamics by a Bayesian Multilayer Perceptron Model for Two Air-Monitoring Sites in Hong Kong, Human and Ecological Risk Assecement, 12, 313

Wang, H. N., Cui, Y. M., Li, L. Y., \& Zhang, H. H. 2008, Solar Flare Forecasting Model Supported with Artificial Neural Network Techniques, Advances in Space Research, 42,1464

Wang, J.-l. 2000, A Two-Pass Neural Network Model for Solar Proton Event Alert, Chinese Astronomy and Astrophysics, 24, 10 